Antarctic Science 33(4), 402-414 (2021) @ The Author(s), 2021. Published by Cambridge University Press. This is an Open Access article, distributed under the terms of the Creative Commons Attribution licence (http://creativecommons.org/licenses/by/4.0/), which permits unrestricted re-use, distribution, and reproduction in any medium, provided the original work is properly cited.

doi:10.1017/S0954102021000262

\title{
Experimental determination of reflectance spectra of Antarctic krill (Euphausia superba) in the Scotia Sea
}

\author{
ANNA BELCHER (1) 1, SOPHIE FIELDING ${ }^{10}$, ANDREW GRAY $\mathbb{1}^{2,3}$, LAUREN BIERMANN $\mathbb{1}^{4}$, \\ GABRIELE STOWASSER (1) ${ }^{1}$, PETER FRETWELL (1) ${ }^{1}$, LOUISE IRELAND (1) ${ }^{1}$ and GERAINT A. TARLING (1) \\ ${ }^{1}$ British Antarctic Survey, Cambridge, CB3 OET, UK \\ ${ }^{2}$ NERC Field Spectroscopy Facility, Edinburgh, EH9 3FE, UK \\ ${ }^{3}$ University of Cambridge, Department of Plant Sciences, Cambridge, CB3 3EA, UK \\ ${ }^{4}$ Plymouth Marine Laboratory, Plymouth, PL1 3DH, UK \\ annbel@bas.ac.uk
}

\begin{abstract}
Antarctic krill are the dominant metazoan in the Southern Ocean in terms of biomass; however, their wide and patchy distribution means that estimates of their biomass are still uncertain. Most currently employed methods do not sample the upper surface layers, yet historical records indicate that large surface swarms can change the water colour. Ocean colour satellites are able to measure the surface ocean synoptically and should theoretically provide a means for detecting and measuring surface krill swarms. Before we can assess the feasibility of remote detection, more must be known about the reflectance spectra of krill. Here, we measure the reflectance spectral signature of Antarctic krill collected in situ from the Scotia Sea and compare it to that of in situ water. Using a spectroradiometer, we measure a strong absorption feature between 500 and $550 \mathrm{~nm}$, which corresponds to the pigment astaxanthin, and high reflectance in the 600-700 nm range due to the krill's red colouration. We find that the spectra of seawater containing krill is significantly different from seawater only. We conclude that it is tractable to detect high-density swarms of krill remotely using platforms such as optical satellites and unmanned aerial vehicles, and further steps to carry out ground-truthing campaigns are now warranted.
\end{abstract}

Received 22 December 2020, accepted 24 May 2021

Key words: Antarctic krill, Euphausia superba, reflectance, remote sensing, South Georgia, South Orkney Islands

\section{Introduction}

Antarctic krill (Euphausia superba) are central to Southern Ocean ecosystems, comprising the highest individual species biomass of any metazoan in the Southern Ocean and forming swarms that can extend over areas of $\sim 100 \mathrm{~km}^{2}$ (Tarling \& Fielding 2016). Krill are an increasingly important commercial target for fisheries, with catch levels managed through international agreement (Nicol 1991). Krill are also an important part of biogeochemical cycles (Schmidt et al. 2011, Belcher et al. 2019, Cavan et al. 2019), particularly due to their swarming behaviour, which results in rapid exploitation of phytoplankton blooms and bulk egestion of rapidly sinking faecal pellets (Belcher et al. 2019). As a result, krill make a strong contribution to the Southern Ocean biological carbon pump, which is tightly coupled to levels of atmospheric carbon dioxide (Kwon et al. 2009). As these episodic pulse events of faecal egestion are difficult to capture with traditional 'snapshot'-style particulate organic carbon (POC) measurements made on oceanographic expeditions, estimates of the carbon exported by krill faecal pellets in the Southern Ocean have, thus far, been mainly addressed through models (Belcher et al. 2019). Present methodological limitations make it difficult to quantify Antarctic krill distribution and biomass and further progress is required in order to manage krill stocks and understand fully their biogeochemical contribution.

Krill are obligate swarmers (Watkins 2000), and although they can sometimes be relatively dispersed, most krill biomass is found within swarms (Tarling et al. 2009, Fielding et al. 2014). The wide circumpolar distribution $\left(\sim 19\right.$ million $\left.\mathrm{km}^{2}\right)$ and the very patchy nature of swarms bring added uncertainty to stock estimates (Atkinson et al. 2009).

The vertical distribution of Antarctic krill is mainly centred around the top $200 \mathrm{~m}$ of the water column (Tarling et al. 2009), although an increasing number of observations indicate that deeper habitats may also be occupied (Clarke \& Tyler 2008, Schmidt et al. 2011). 


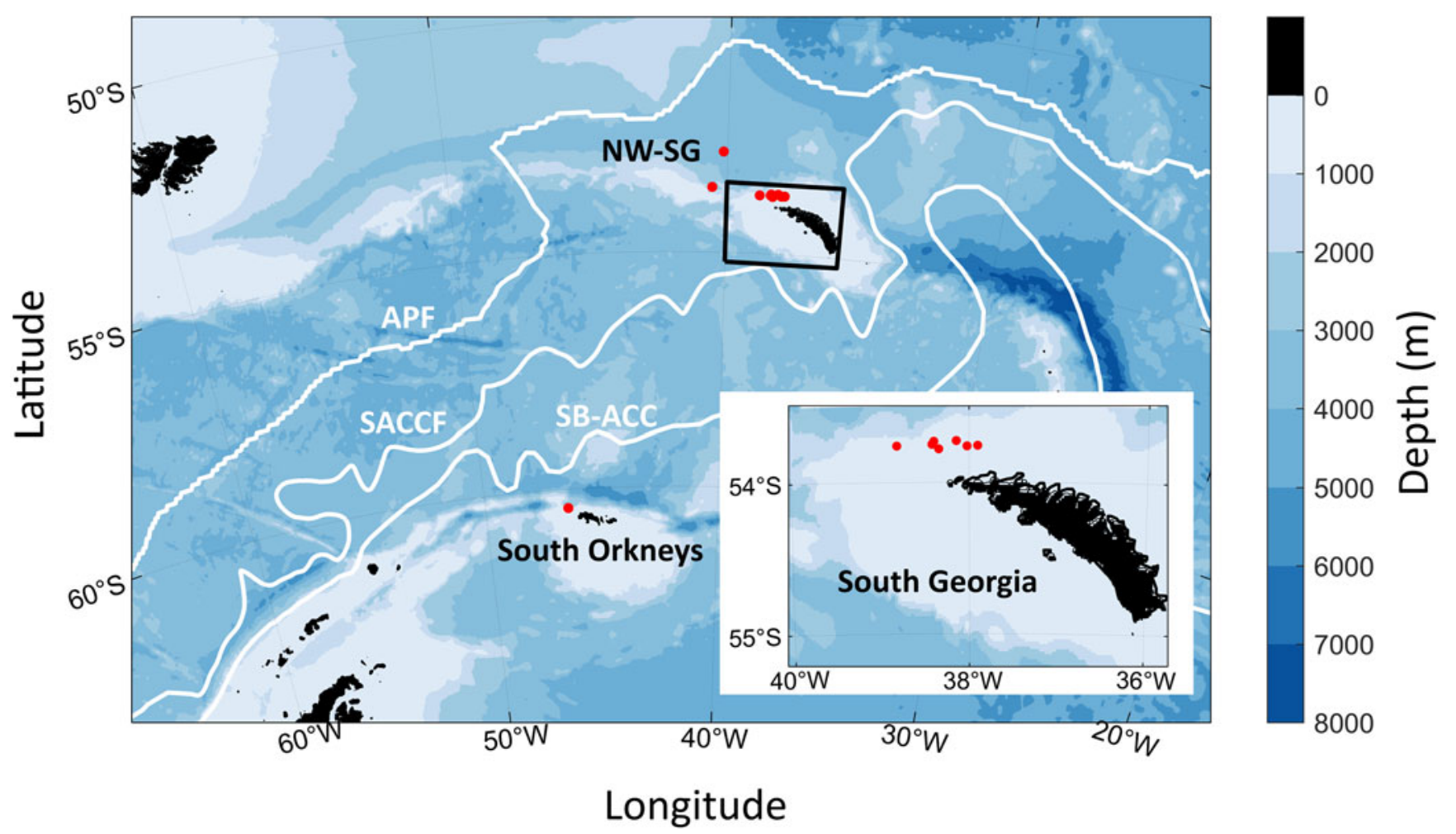

Fig. 1. Location of RMT8 deployments to sample krill for reflectance measurements (red circles). White lines are mean frontal positions of the Antarctic Polar Front (APF), the Southern Antarctic Circumpolar Current Front (SACCF) and the Southern

Boundary-Antarctic Circumpolar Current (SB-ACC). Sampling areas, South Orkneys, South Georgia and north-west South Georgia (NW-SG) are labelled.

The near-surface ( $0-15 \mathrm{~m}$ water depth) distribution of krill is less described, probably related to the methodological challenge of sampling there. Traditional krill survey methods utilize nets towed obliquely, which suffer from problems of net avoidance (Everson \& Bone 1986), particularly during the day and close to the surface (Simard \& Sourisseau 2009). The alternative method for estimating krill density uses echo-sounders to provide wider spatial scale measurements. However, hull-mounted transducers are unable to sample the near-surface layer (upper $\sim 15 \mathrm{~m}$ ) due to near-field effects (Tichy et al. 2003). We know from historical records and verbal descriptions that krill can swarm at the surface; for example, Marr (1962) summarizes, 'so then as can be seen from the decks of vessels in daylight the krill are manifestly concentrated in dense patches on or very close to the surface sea'. Our estimates of krill biomass and distribution are missing this component, which limits the accuracy of krill density assessments and our ability to monitor changes over time (Atkinson et al. 2019). Accurate assessments of near-surface swarms are vital both for sustainable fisheries management and for accurate quantification of carbon export and sequestration in the Southern Ocean.

The fishery for Antarctic krill is the largest by tonnage in the Southern Ocean (Nicol \& Foster 2016) and is managed by the Conservation of Antarctic Marine Living Resources (CCAMLR). Catch limits are informed by large-scale international krill surveys, such as the CCAMLR-2000 survey (Hewitt et al. 2004) and, more recently, the International Synoptic Krill Survey (Macaulay et al. 2019). Surveys at the spatial scale of the South Atlantic take many ships a month to complete and, as a result, have only been undertaken rarely, on average around every 20 years since the 1980s. Variability in krill populations, seasonally with krill recruitment and on longer timescales with changing environmental conditions (Atkinson et al. 2019), necessitates more frequent biomass surveys beyond the local scale in which they are currently undertaken (Fielding et al. 2014).

Satellites offer a way of collecting synoptic data over large spatial scales and, with continued improvements in spatial, temporal and spectral resolution, have been used to assess populations of whales (e.g. Cubaynes et al. 2019) and, more recently, copepods (Basedow et al. 2019). Davies et al. (2021) found that large coloured particles can impact remote sensing reflectance signals, suggesting that there is scope to explore the application of remote sensing to the monitoring of swarming. Additionally, the pigment astaxanthin, which is a dominant carotenoid pigment in krill (e.g. Czerpak et al. 1980), has been used to indicate a 


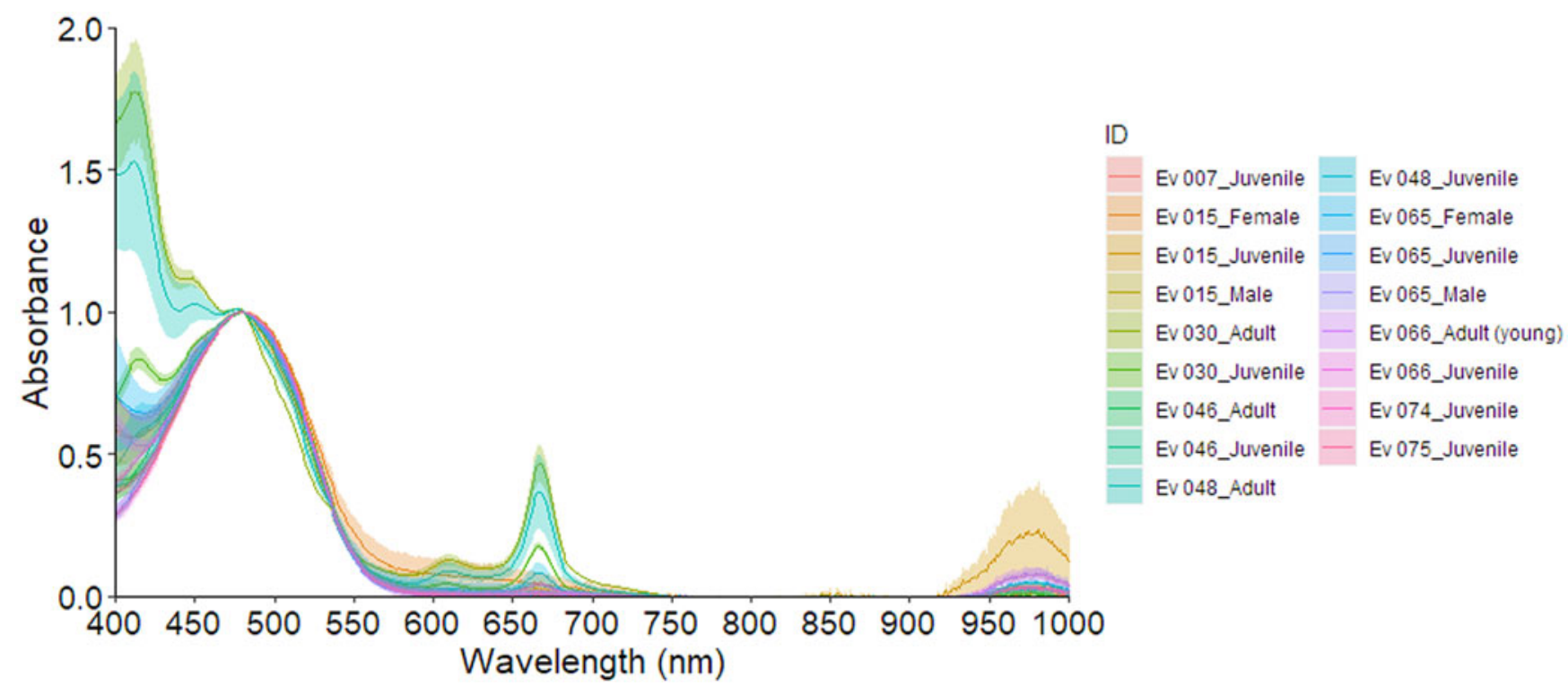

Fig. 2. Absorbance spectra (from 400 to $1000 \mathrm{~nm}$ ) of krill extracted in 100\% acetone for all sites: South Georgia shelf (Ev 015-066), South Orkney Islands (Ev 007) and north-west South Georgia (Ev 074-075). All absorbances have been corrected for turbidity at $750 \mathrm{~nm}$ and normalized to an absorbance of 1 at $480 \mathrm{~nm}$. Data are coloured by the net haul ID (event) at each station with shading denoting the standard deviation across measurements made on multiple krill from that event.

krill-rich diet when examining the reflectance spectra of penguin guano (Rees et al. 2017).

Remote detection relies on sufficient densities of krill spending time in the upper $10 \mathrm{~m}$ of the water column and over sufficient spatial scales for the resolution of the satellite sensor. Cloud cover also presents an issue in the Southern Ocean. Before the possibility of remote detection can be assessed, spectral reflectance measurements of krill collected in situ are required. The pigmentation of krill is affected by their age, health and the food they eat (Auerswald et al. 2008), and thus freshly caught krill from their natural environment are required for such measurements. In this study, we make the first measurements of the spectral reflectance of krill caught in situ in the Scotia Sea, Southern Ocean, a region with notably high concentrations of krill (Atkinson et al. 2009). We examine the spectral signature of krill collected at various locations in the Scotia Sea to determine whether the spectra are sufficiently different from that of ocean water. These measurements allow us to assess whether it is tractable to differentiate the signal of a krill swarm from that of open ocean water within suitable satellite imagery.

\section{Methods}

Net sampling

Krill reflectance experiments were conducted aboard the RRS James Clark Ross during research cruise JR19001 to the Scotia Sea in the Southern Ocean (14 November 2019-26 December 2019). Krill were sampled from three main regions (Fig. 1): South Orkney Islands (SOI), South Georgia shelf (SG-shelf) and north-west South Georgia (NW-SG); SOI and SG-shelf in particular are regions known for high krill concentrations. Krill swarms were located using a SIMRAD EK60 multi-frequency echosounder $(38,70,120$ and $200 \mathrm{kHz})$. Krill swarms of at least $100 \mathrm{~m}$ in length and occurring in the top $10-100 \mathrm{~m}$ were targeted to ensure success of capture. A Rectangular Midwater Trawl (RMT8; Everson \& Bone 1986) with remote opening and closing ensured only the krill swarm was targeted. Once on board, krill were separated into different sex-stage categories, firstly to distinguish adults from juveniles and, where sufficient numbers allowed, then into males and females. Krill were stored in buckets of underway seawater $(0-4 \mathrm{~h})$ in a temperature-controlled room at $4^{\circ} \mathrm{C}$ until analysis.

\section{Pigment extraction}

Immediately after krill samples were brought on board and before spectral measurements were made, three krill were taken for each krill sex-stage category for pigment extraction. Individual krill were homogenized in glass vials in $5 \mathrm{ml}$ of $100 \%$ acetone using an electric homogenizer. The vials were left in the dark at $-20^{\circ} \mathrm{C}$ for $24 \mathrm{~h}$. After this period, the extract was carefully pipetted out (to avoid particulates) into a $1 \mathrm{~cm}$ cuvette. The absorbance of each sample was analysed on a Cary60 UV-Vis spectrophotometer. A blank reading and a baseline reading from 190 to $1600 \mathrm{~nm}$ were taken using $100 \%$ acetone. To correct for turbidity, the absorbance value at $750 \mathrm{~nm}$ was 


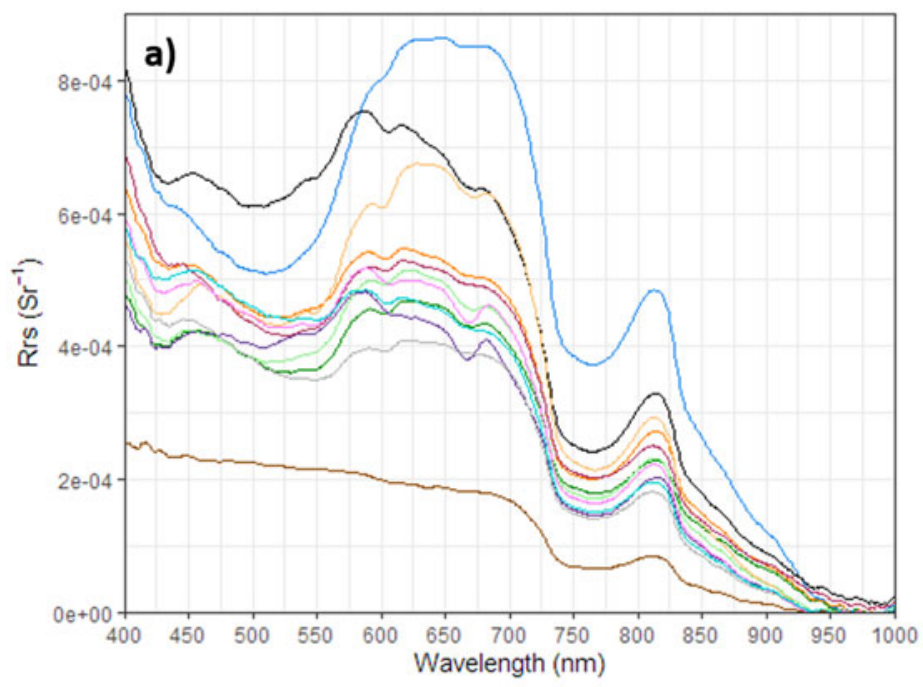

ID

— Ev 007_Juvenile (150)

- Ev 030_Adult (50)

- Ev 030_Juvenile (60)

— Ev 046_Adult (60)

- Ev 046_Juvenile (70)

- Ev 065_Female (70)

- Ev 065_Male (70)

- Ev 066_Adult (young) (70)

- Ev 066_Juvenile (90)

- Ev 074_Juvenile (90)

— Ev 075_Juvenile (90)

- FSW

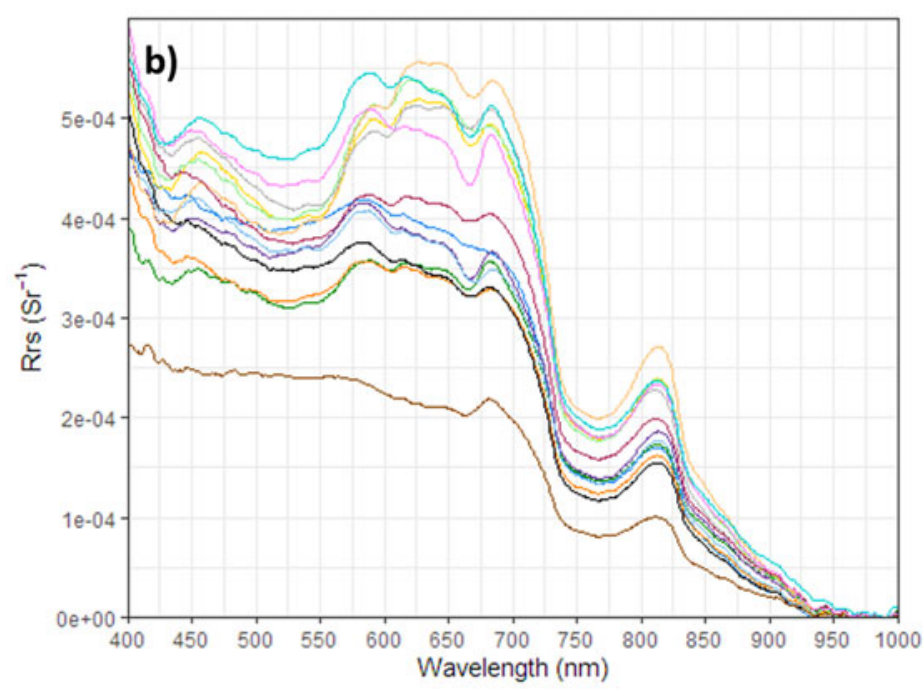

ID

- Ev 007_Juvenile (100)

- Ev 015_019_Adult (70)

- Ev 030_Adult (60)

- Ev030_Juvenile (60)

- Ev 046_Adult (50)

- Ev 046_Juvenile (70)

- Ev 048_Adult (70)

- Ev 048_Juvenile (70)

- Ev 065_Female (70)

- Ev 065_Male (70)

- Ev 066_Adult (young) (70)

- Ev 066_Juvenile (90)

- Ev 074_Juvenile (90)

- Ev 075_Juvenile (90)

- UsW

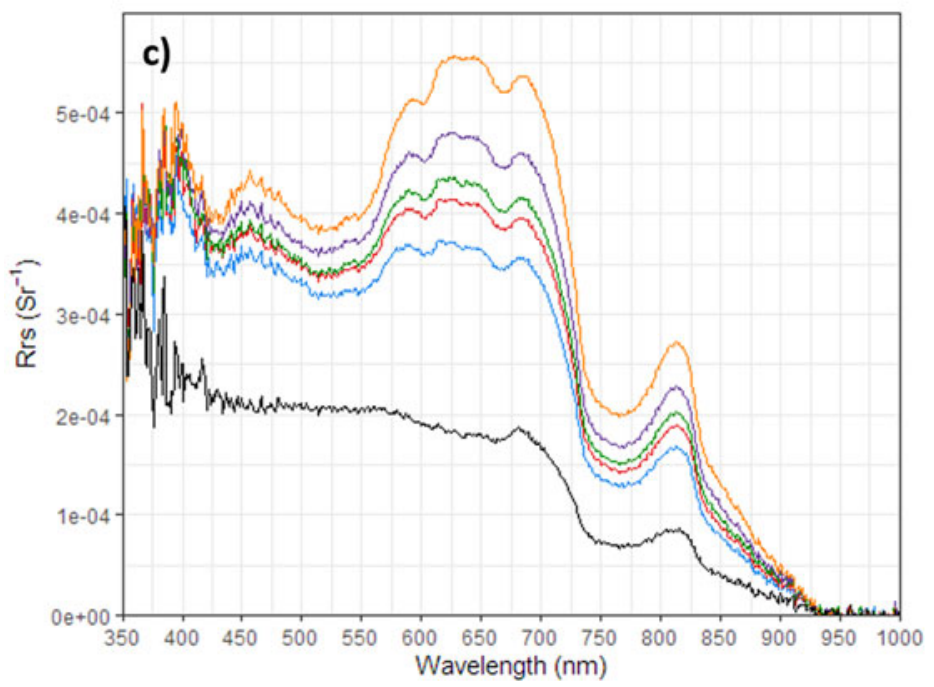

$$
\begin{aligned}
& \text { Krill_count } \\
& -40 \text { krill } \\
& -50 \text { krill } \\
& -60 \text { krill } \\
& -70 \text { krill } \\
& -80 \text { krill } \\
& -\quad \text { USW }
\end{aligned}
$$

Fig. 3. $\mathrm{R}_{\mathrm{rs}}\left(\mathrm{Sr}^{-1}\right)$ from reflectance experiments for wavelengths from 400 to $1000 \mathrm{~nm}$. Experiments carried out in a. filtered seawater (FSW) and b. unfiltered seawater (USW). Data are for the maximum number of krill added at each station (30-150, number given in parentheses in legend). Line colours relate to the experiment ID code. Data have been smoothed (Savitzky-Golay smoothing, polynomial 3, filter length 15). c. $R_{r s}$ profiles of male krill sampled from the South Georgia shelf (event 065). The total $R_{r s}$ of the krill and water is shown for each addition of krill, ranging from 40 to 80 krill individuals in the bucket. 
Table I. Details of samples collected and experiments undertaken during research cruise JR19001 in our three regions: South Orkney Islands (SOI), South Georgia shelf (SG-shelf) and north-west South Georgia (NW-SG). The maximum number of krill added to each reflectance experiment is shown for filtered seawater (FSW) and unfiltered seawater (USW), where numbers were different for the sex stage of krill.

\begin{tabular}{|c|c|c|c|c|c|c|c|c|c|}
\hline \multirow[b]{2}{*}{ Time (GMT) } & \multirow[b]{2}{*}{$\begin{array}{l}\text { Latitude } \\
\left({ }^{\circ} \mathrm{N}\right)\end{array}$} & \multirow[b]{2}{*}{$\begin{array}{l}\text { Longitude } \\
\left({ }^{\circ} \mathrm{E}\right)\end{array}$} & \multirow[b]{2}{*}{ Region } & \multirow[b]{2}{*}{$\begin{array}{c}\text { Event } \\
\text { number }\end{array}$} & \multirow[b]{2}{*}{$\begin{array}{l}\text { Krill sex-stage } \\
\text { sampled }\end{array}$} & \multirow[b]{2}{*}{$\begin{array}{l}\text { Acetone } \\
\text { extraction }\end{array}$} & \multirow[b]{2}{*}{$\begin{array}{l}\text { Reflectance } \\
\text { experiment }\end{array}$} & \multicolumn{2}{|c|}{$\begin{array}{l}\text { Maximum krill } \\
\text { added }\end{array}$} \\
\hline & & & & & & & & FSW & USW \\
\hline $\begin{array}{l}\text { 13 December 2019, } \\
\text { 03:02 }\end{array}$ & -53.5901 & -40.4784 & NW-SG & 075 & $\mathbf{J}$ & Yes & Yes & 90 & 90 \\
\hline $\begin{array}{l}\text { 12 December 2019, } \\
20: 47\end{array}$ & -52.8300 & -40.1391 & NW-SG & 074 & $\mathrm{~J}$ & Yes & Yes & 90 & 90 \\
\hline $\begin{array}{l}\text { 11 December 2019, } \\
\text { 01:35 }\end{array}$ & -53.7393 & -38.1479 & SG-shelf & 065 & Female, male & Yes & Yes & 70 & 70 \\
\hline $\begin{array}{l}8 \text { December 2019, } \\
\text { 05:23 }\end{array}$ & -53.7200 & -38.4086 & SG-shelf & 048 & $\mathrm{~J}, \mathrm{~A}$ & Yes & Yes & - & 70 \\
\hline $\begin{array}{l}8 \text { December 2019, } \\
00: 39\end{array}$ & -53.7446 & -37.9203 & SG-shelf & 046 & $\mathrm{~J}, \mathrm{~A}$ & Yes & Yes & $\begin{array}{c}70(\mathrm{~J}), 60 \\
\text { (A) }\end{array}$ & $\begin{array}{l}70 \text { (J), } 50 \\
\text { (A) }\end{array}$ \\
\hline $\begin{array}{l}2 \text { December 2019, } \\
\text { 20:00 }\end{array}$ & -53.7674 & -38.0254 & SG-shelf & 015 & $\mathrm{~J}$, male, female & Yes & Yes & - & \\
\hline $\begin{array}{l}24 \text { November } 2019 \text {, } \\
18: 30\end{array}$ & -60.3936 & -46.5036 & SOI & 007 & $\mathrm{~J}$ & Yes & Yes & 150 & 100 \\
\hline
\end{tabular}

$\mathrm{A}=$ adult; $\mathrm{J}=$ juvenile.

subtracted for each wavelength (Standing Committee of Analysts 1983) based on the assumption of zero absorption at this wavelength (i.e. the measured signal was solely due to particle scattering). Additionally, as the masses of individual krill homogenized were not identical, to compare the spectral shapes of each extraction, we normalized our absorbance measurements to the absorbance at $480 \mathrm{~nm}$ (thus setting absorbance at $480 \mathrm{~nm}=1$ ), where a consistent and defined peak was observed in the spectra.

\section{Spectral measurements}

To help determine whether it might be possible to distinguish the signal of a krill swarm from that of open water, we made on-board measurements of the reflectance spectra of krill. Once samples had been taken for pigment extraction, reflectance spectra of multiple krill (from each krill sex-stage category) were recorded using artificial illumination in a dark room using an ASD FieldSpec 4 Spectroradiometer. This instrument detects radiation in the range $350-2500 \mathrm{~nm}$ with a full width at half-maximum (FWHM) of $3 \mathrm{~nm}$ in the visible range $(350-700 \mathrm{~nm})$. Measurements were recorded in a matt black bucket (121), with average reflectance of 3.1\% within the visible region of the spectrum. The bare fibre fore-optic of the FieldSpec 4 was used, giving a field of view of $\sim 7 \mathrm{~cm}^{2}$ at mid-full bucket depth in water. All measurements were taken under a 70 watt quartz- tungsten-halogen lamp (ASD Illuminator Reflectance lamp) with no other background light. A skylight blocked approach, adapted from Lee et al. (2019), was used to calculate remote sensing reflectance $\left(R_{r}\right)$. A reference reading was taken at the start and end of each experiment using a $20 \%$ grey Spectralon $\AA$ panel (Labsphere, Inc.), which had been calibrated against a reference Spectralon ${ }^{\circledR}$ panel, applying the $8 \% T$ method used by the Natural Environment Research Council (NERC) Field Spectroscopy Facility. The optimal integration time was also set against this $20 \%$ panel, giving an in-water signal-to-noise ratio of between 15 and 170 across the visible region. The spectroradiometer's entrance optics were secured to a tripod and measurements made at nadir (pointing vertically down to the centre of the bucket). The fibre-optic end was positioned just below the surface of the water when measuring the spectra of krill or water, removing the effects of surface glint measurements. Reflectance was recorded for each krill sex-stage category in in situ seawater, both $0.2 \mu \mathrm{m}$ filtered seawater (FSW) and unfiltered seawater (USW; water sourced from the ship's underway system with intake at a depth of $5 \mathrm{~m}$ ). Initially, the reflectance of only USW or FSW was measured $\left(R_{r}\right.$, water $)$, following which krill were added sequentially (from 5 to 100 krill) with a reflectance measurement made at each addition $\left(R_{r}\right.$, total $)$. All added krill were alive and the majority were active but not freely swimming throughout the full depth 


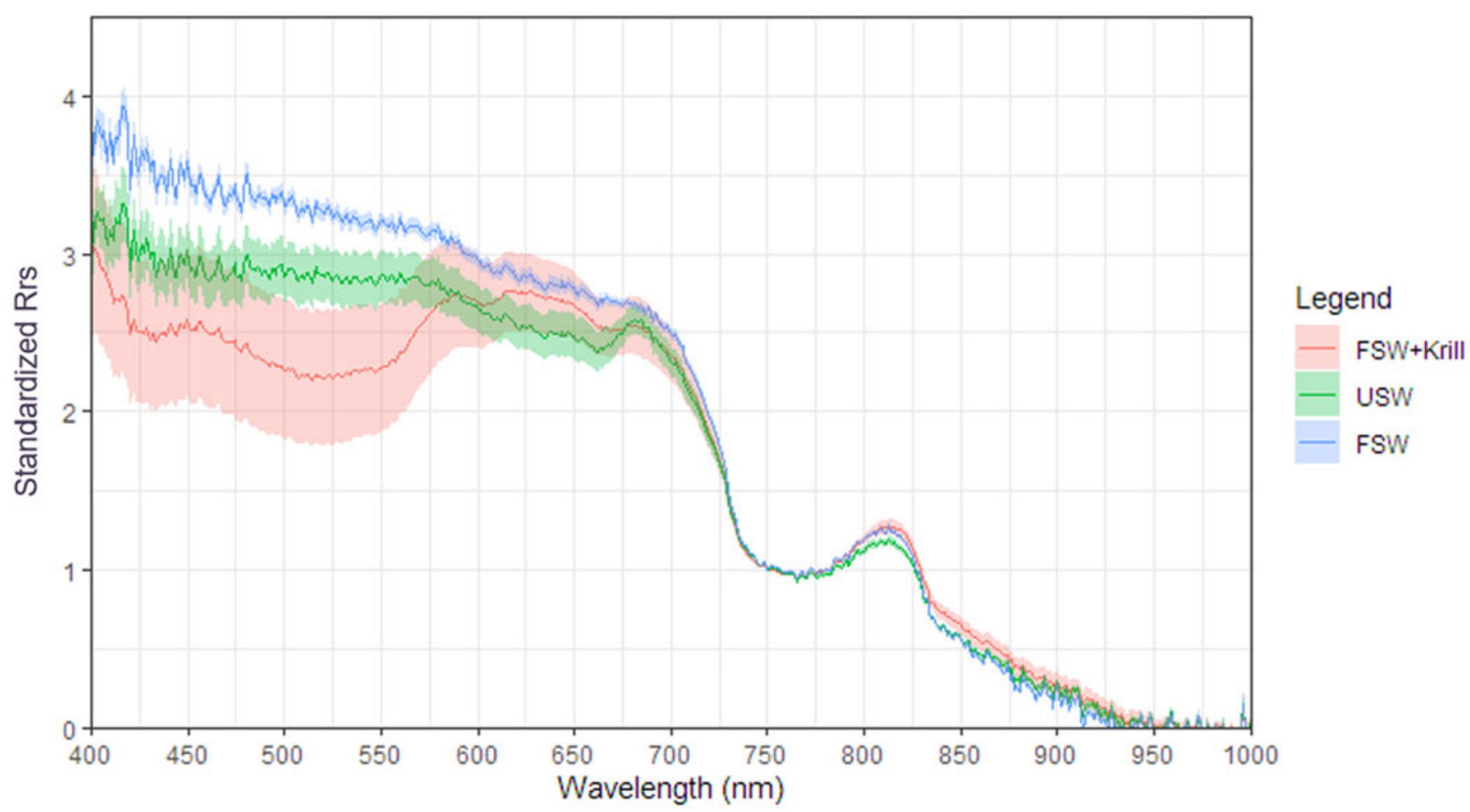

Fig. 4. Mean $\mathrm{R}_{\mathrm{rs}}$ profiles of krill in filtered seawater (FSW+Krill; red) and both filtered seawater (FSW; blue) and unfiltered seawater (USW; green) from reflectance experiments standardized to $750 \mathrm{~nm}$. Data are for wavelengths from 400 to $1000 \mathrm{~nm}$. Shading shows the standard deviation based on individual experiments shown in Fig. 3.

of the incubation apparatus; $\sim 10 \%$ were freely swimming in random directions. A total of 10 reflectance spectra were collected for each addition of krill, with each of these spectra representing an average of 26 readings from the spectroradiometer. The mean of these 10 profiles was calculated to give the raw reflectance measurement (R). The maximum number of krill added was based on the availability of healthy krill at each station sampled. A total of 10 stations were sampled, and the reflectance of krill separated into adults and juveniles was measured at each station. For some of these stations, sufficient numbers made it possible to separate adults further into males and females and to run separate experiments on these categories.

Remote sensing reflectance $\left(\mathrm{R}_{\mathrm{rs}}\right)$ was calculated for each wavelength $(\lambda)$ using the following equation:

$$
\mathrm{R}_{\mathrm{rs}}(\lambda)=\frac{\mathrm{R}(\lambda)}{\pi \mathrm{E}(\lambda)} \mathrm{k}
$$

where $\mathrm{E}$ is the reference reflectance from the $20 \%$ Spectralon panel (mean of start and end measurements and $\mathrm{R}$ is the raw reflectance measurement. $\mathrm{k}$ is the Spectralon panel's calibrated reflectance, which, along with $\pi$, converts $\mathrm{E}$ from reflected radiance to irradiance. $\mathrm{R}_{\mathrm{rs}}$ varied with the number/size of krill. Therefore, to compare better the spectral shape between experiments and to correct for scattering effects (Mobley 1999), we normalized $\mathrm{R}_{\mathrm{rs}}$ measurements to the reflectance at $750 \mathrm{~nm}$ (setting $R_{\mathrm{rs}}$ at $750 \mathrm{~nm}=1$ ). The value of $750 \mathrm{~nm}$ corresponded to where the total reflectance signal was dominated by a relatively constant water absorption feature.

\section{Contact probe}

Reflectance measurements were also taken with an ASD contact probe attached as the fore-optic to the ASD FieldSpec 4 . Three to five krill from each category were placed on a matt black tray and the reflectance spectra measured by holding the probe against the krill. As with the reflectance experiments in the bucket, a reference reading was taken of a white Spectralon panel, as well as a measurement of the black tray only. However, data collected were not reliable as the sensor fogged up due to the moisture from the krill and the heat of the lamp. These data were therefore not analysed further.

\section{Results}

\section{Pigment extractions}

A defined peak in absorbance at $480 \mathrm{~nm}$ was observed for all krill extractions (Fig. 2), with an additional clear peak 

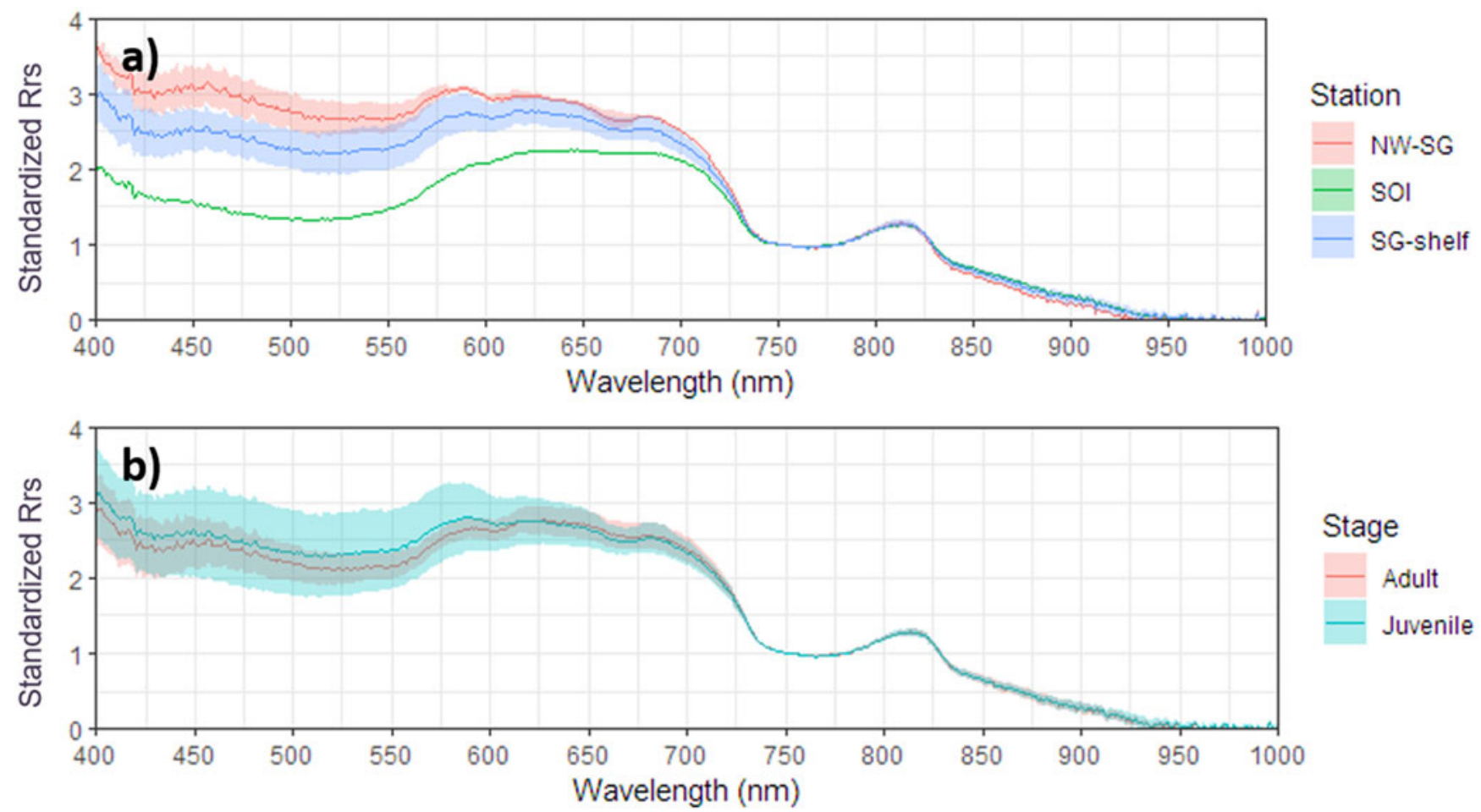

Fig. 5. a. Mean $\mathrm{R}_{\mathrm{rs}}$ profiles of krill in filtered seawater (FSW), calculated for a. station group: north-west South Georgia (NW-SG; red), South Orkney Islands (SOI; green), South Georgia shelf (SG-shelf; blue); and b. stage: adult (red), juvenile (turquoise). Data for wavelengths from 400 to $1000 \mathrm{~nm}$ and have been standardized to $750 \mathrm{~nm}$. Shading shows the standard deviation based on individual experiments shown in Fig. 3.

at $670 \mathrm{~nm}$ for samples from the SG-shelf site. Additionally, we see quite a lot of variability in the region $<450 \mathrm{~nm}$, with multiple different peaks and high absorbance regions.

\section{Krill reflectance spectra}

We conducted 14 successful reflectance experiments from 10 different stations (Fig. 3 \& Table I). The maximum $\mathrm{R}_{\mathrm{rs}}$ measured during each experiment was quite low at $<0.001 \mathrm{Sr}^{-1}$, and this varied between experiments relating to the size, number and position of krill in the bucket. The number of krill in the bucket has a strong impact on the magnitude of $\mathrm{R}_{\mathrm{rs}}$ (Fig. 3c). We compare here the shape of the spectra, as it is the spectral shape that we are interested in for determining whether krill have a significantly different spectral reflectance from water (and other ocean properties; e.g. chlorophyll) to enable remote detection. We present the data for the maximum number of krill added to each experiment in order to show the strongest reflectance signal. Mean $\mathrm{R}_{\mathrm{rs}}$ profiles (of both krill and USW) for all experiments are shown in Fig. 4.

Reflectance spectra collected from these bucket experiments may be used to generate a spectral 'signature' that could assist with identification of krill remotely. For this to be possible, we need to determine whether there are reflectance peaks or absorbance troughs unique to krill and notably different from USW or FSW. In the USW, there is a small peak present at $\sim 680 \mathrm{~nm}$, which is removed through filtration (Fig. 3a \& b). This peak is also consistently present in the krill sampled in USW, with the exception of the highest concentrations of juveniles Ev 007 (Fig. 3b). With regards to the krill sampled in FSW, however, this $680 \mathrm{~nm}$ peak is not consistently present (Fig. 3a). For future remote sensing-based detection approaches, reflectance information at $680 \mathrm{~nm}$ (far red to near infrared) is thus probably less robust for krill detection. The reflectance peak in the near infrared at $\sim 810 \mathrm{~nm}$ persists in both the USW and FSW (without krill), making the information in these wavelengths also less unique to krill and thus unsuitable for detection. The three reflectance peaks of interest for krill detection are at $450-460 \mathrm{~nm}$ (in the violet range of light), at $\sim 580-590 \mathrm{~nm}$ (yellow light) and at $625 \mathrm{~nm}$ (orange light). These three peaks are not present in FSW and/or USW, and they appear to present consistently with the exception of the experiment with 150 juvenile krill from Ev 007 in FSW.

We normalize reflectance values to the reflectance at $750 \mathrm{~nm}$ (Fig. 4) to remove effects of varying magnitude between krill, USW and FSW spectra and to focus on 
the differences in spectral shape. This highlights that the spectral shape was very similar across the different experiments containing krill sampled from different regions. Samples containing krill show high reflectance in the red $(600-700 \mathrm{~nm})$ part of the spectrum, giving the krill its orange-pink colour. Comparing the spectra of water only and water containing krill, for krill we see a clear, strong absorption feature between $\sim 460$ and $590 \mathrm{~nm}$, with the strongest absorption between 500 and $550 \mathrm{~nm}$. We also see a narrow absorption feature at $\sim 430 \mathrm{~nm}$. Additionally, there is a small absorption feature at $655-675 \mathrm{~nm}$ in both the krill and USW spectra. All spectra show absorption between 700 and $810 \mathrm{~nm}$.

Comparing further the individual experiments, we calculate the mean of normalized reflectance spectra for each station group (SOI, $n=1$; SG-shelf, $n=11$; NW-SG, $n=2$ ), as well as each krill stage (adult, $n=7$; juvenile, $n=7$ ) (Fig. 5). Unfortunately, we do not have sufficient replicates of male and female krill to separate these, so we group them into the adult category. However, the small standard deviation of adult krill highlights that male and female adult krill had similar spectra (Fig. 5b). The proximal stations (NW-SG and SG-shelf) show similar spectra. Krill caught from SOI show some minor differences to the SG krill: notably, there being no absorbance feature at $\sim 670 \mathrm{~nm}$. There is considerable overlap between the spectra of adult and juvenile krill with the same broad absorption feature between $\sim 460$ and $590 \mathrm{~nm}$ (Fig. 5b). There is higher variability between the spectra of juvenile krill, shown by the higher standard deviations at wavelengths $<700 \mathrm{~nm}$.

\section{Discussion}

In order to assess whether it might be feasible to detect krill remotely, it is first necessary to measure the reflectance spectra of fresh krill caught in situ to ensure natural feeding conditions (diet affects pigmentation; Siegel 2016) and other physiological and environmental factors (Auerswald et al. 2008) are maintained in order to retain natural reflectance properties. Here, we present the first such measurements and discuss the shapes of the obtained spectra.

\section{Reflectance properties of krill}

Our experiments highlight that freshly caught krill show strong absorbance in the $460-590 \mathrm{~nm}$ range, with strongest absorption between 500 and $550 \mathrm{~nm}$. This agrees quite well with the peak in absorption at $480 \mathrm{~nm}$ that we measured from the extraction of fresh krill in $100 \%$ acetone (Fig. 2). It also corresponds well with the absorbance peak of astaxanthin, which is known to be present in krill (e.g. Auerswald et al. 2008). It is thus probable that absorbance in the $460-590 \mathrm{~nm}$ range is at least in part due to the presence of astaxanthin and other carotenoids (astaxanthin being the most dominant carotenoid pigment in krill; e.g. Czerpak et al. 1980). As expected from the red colouration of krill, reflectance is higher in the $600-700 \mathrm{~nm}$ range (corresponding to red) than in blue/green wavelengths $(<600 \mathrm{~nm})$. This pattern was true for all stages of krill sampled and at all stations, suggesting that these features are likely to be true for all krill populations. The three reflectance peaks at $\sim 450-460 \mathrm{~nm}$ (violet), $580-590 \mathrm{~nm}$ (yellow) and $625 \mathrm{~nm}$ (orange) are not present in seawater and may be sufficiently unique to krill to be used for future satellite or drone-based detection algorithms. The Copernicus Programme's Sentinel-2 and Sentinel-3 optical satellites do not specifically collect data at $580-590 \mathrm{~nm}$, but DigitalGlobe's WorldView-2 and WorldView-3 satellites (launched in 2009 and 2014, respectively) do, and at high spatial resolution.

A number of other pelagic crustacean zooplankton (e.g. copepods, mysids, decapods) contain carotenoid pigments within their exoskeletons. However, in the Antarctic Southern Ocean, pelagic crustaceans do not exhibit the tendency to swarm within the surface layers at scales resolvable from remote sensing, which we consider to be a unique feature of Antarctic krill. In the Arctic, Basedow et al. (2019) did report on the satellite detection of surface slicks of copepods, but these are likely to be a result of a specific sets of environmental conditions (upwelling and unusual salinity gradients affecting buoyancy), which we do not consider to be a widespread phenomenon in the Southern Ocean.

Some experiments in the SG-shelf region show an absorbance feature at $670 \mathrm{~nm}$ (Fig. 3), corresponding well to the absorbance peak seen in acetone extractions (Fig. 2). This probably relates to chlorophyll $a$ in the gut of the krill and highlights the potential challenge of distinguishing krill containing gut chlorophyll from phytoplankton-rich waters. The presence of chlorophyll pigments in the gut is supported by comparison of USW and FSW (Fig. 4). The USW also shows a peak at $670 \mathrm{~nm}$ whereas FSW does not, highlighting the presence of chlorophyll-containing algae in the water that may be grazed on by krill.

Diatoms (Dinophyta) and Cryptophyta are dominant phytoplankton taxa in South Georgia and the South Orkney Islands, respectively (Korb et al. 2012, Nunes et al. 2019). Reflectance simulated from absorption spectra measured on cultures in the lab (e.g. Xi et al. 2017) as well as direct measurements (e.g. Soja-Wozniak et al. 2018) made on the main phytoplankton groups (Chlorophyta, Cyanophyta, Haptophyta, Dinophyta, Cryptophyta and Heterkontophyta) suggest that absorption by these phytoplankton groups is strongest between $\sim 520$ and $650 \mathrm{~nm}$. Gut phytoplankton may therefore contribute to 
part of the absorption feature we measured between 460 and $590 \mathrm{~nm}$. However, if the gut contents were affecting the reflectance spectra greatly, we would expect to see a large difference in spectra between the regions sampled, as the phytoplankton and thus feeding conditions are different. The fact that we see a consistent broad absorbance feature within each of the three regions sampled therefore gives confidence in the potential to distinguish krill.

Overall, the exact composition of dietary algae as well as gut fullness will affect the reflectance spectra and thus will cause variability between krill individuals sampled in different regions. It will therefore probably be necessary to isolate regions of the reflectance spectrum that are not impacted by phytoplankton pigments and use ratios between multiple wavelengths to distinguish krill.

\section{Experimental considerations}

In assessing the feasibility of using the features identified by our reflectance measurements for the remote detection of krill, it is important to consider the experimental uncertainties of our data and the limitations of remote sensing. Our experiments on fresh krill were carried out on board the RRS James Clark Ross in laboratory conditions and thus differ from those observed by satellites in several ways. Factors that need to be considered include the light source, bucket volume, influence of the atmosphere, signal-to-noise ratios, packing concentrations, depth and size of krill swarms and confounding optical properties. We address these each in turn.

As a light source for our experiments, we used an ASD quartz-tungsten-halogen lamp. This provides a stable light field across a similar spectral range to natural sunlight (ASD, Inc. 2010) to allow for accurate calibrations with the Spectralon panel to be carried out. However, this is not equivalent to natural conditions, where brightness can vary with cloud cover. As cloud cover prevents the acquisition of ocean colour data from satellites and as it is possible to mask cloud-covered and high-glint areas, the usable data from satellites should be similar to those of our experimental light field. Additionally, by using a ratio of wavelengths, it is only the relative magnitudes that are important.

For practical reasons in terms of both space and the number of krill that were required, it was not possible to conduct the experiments in a very large aquarium to negate any possible impact of the container on the spectral signature measured. A matt black bucket with low, flat reflectance (shown in Supplemental Fig. S1) was chosen to minimize container effects upon water-leaving radiance. Additionally, the experimental setup ensured a small enough field of view that the sides of the bucket were not in range. By comparing the signal of water only in the bucket $\left(\mathrm{R}_{\mathrm{r} \text {, water }}\right)$ and that with krill also $\left(\mathrm{R}_{\mathrm{r}, \text { total }}\right)$, even if the bucket impacted our measurements, we can still attribute differences between these spectra to the presence of krill. It is important to note that there may be some influence of the bucket in the $R_{r}$, total spectra, and thus spectra obtained remotely of in situ krill in ocean water will differ.

Our on-board experiments were measuring krill at close range, just below the surface $(\sim 12.5-25.0 \mathrm{~cm})$, and are thus assumed to be free from atmospheric effects and bidirectional effects at the air-water interface. Satellites measure the radiance at the top of the atmosphere (TOA), so the contribution of the atmosphere and sun glint (reflection of direct sunlight off the sea surface) need to be accounted for to estimate the water-leaving reflectance. In clear open ocean (Case-1) waters, the contribution of the atmospheric path radiance to the total TOA radiance is $\sim 90 \%$ in the blue and green and higher in the red (IOCCG 2010). This is primarily due to molecular and aerosol scattering. Satellite-measured radiance can be atmospherically corrected; for example, Sentinel-3 level-2 data are atmospherically corrected using a multiple scattering algorithm/radiative transfer approach. Our measured reflectance spectra should therefore be comparable to these data. However, as these corrections involve aerosol models and are imperfect corrections, it is not possible to compare absolute reflectance values between in situ and satellite remote sensing reflectance data.

The reflectance values we measured for krill in seawater were low, highlighting the strong absorbance of light in water, which is greater towards the red end of the spectrum. Therefore, the signal-to-noise ratio of any remote sensor must be adequate to detect these small shifts in reflectance.

For a krill swarm to be detectable remotely, the packing concentration, depth and size of the krill swarms must be considered. The aim of our experiments was to measure the spectral signature of krill and so individuals were added sequentially to obtain a sufficiently strong reflectance signal (high enough signal-to-noise ratio). We present here the data for the maximum number of krill added for each experiment, which differed depending on the availability of krill in a suitable condition for experiments. The maximum numbers added ranged from 30 to $150 \mathrm{krill}$, representing packing concentrations of 2500-12,500 ind. $\mathrm{m}^{-3}$. The range of packing concentrations reported in the literature is large, leading to uncertainty as to what is a 'typical' packing concentration of a krill swarm. The RMT8 net deployments during JR19001 aimed to target the periphery of krill swarms so as not to overload the net; we found packing concentrations of up to 22 ind. $\mathrm{m}^{-3}$. Acoustic-based estimates have a large range; Fielding et al. (2012) found a typical range in packing concentrations in the Scotia Sea in spring of $3-81$ ind. $\mathrm{m}^{-3}$, but with concentrations 
reaching $\sim 1650$ ind. $\mathrm{m}^{-3}$. Conversely visual observations exceed these estimates by two orders of magnitude, with estimates ranging from 25,000 to 64,000 ind. $\mathrm{m}^{-3}$ (Hamner \& Hamner 2000 and references therein). Considering the irregular and, at times, indented and filamentous structure of krill swarms, it is not surprising that packing concentrations are not uniformly high (Ryabov \& Tarling 2019) and may additionally vary with proximity to land (Klevjer et al. 2010).

Sufficient packing concentrations are required for detection using satellites; however, the exact concentration needed will also depend on the depth of the swarm. Ocean colour observations are limited to the first optical depth (the depth to which satellites can measure in-water properties; Dierssen \& Randolph 2013) and therefore krill swarms sitting deeper than this will not be detected. The optical constituents of the water (e.g. coloured dissolved organic matter, particle concentration) affect the exact optical depth but, with optically clear waters, we would not expect to see below $\sim 15 \mathrm{~m}$ depth. Within this surface layer, the deeper the swarm, the higher the packing concentration that would be needed for detection. The depth at which krill reside can vary on a diel basis, although coordinated diel vertical migration seems more pronounced in younger developmental stages (Tarling et al. 2018, Cavan et al. 2019). There is evidence to suggest that the vertical distribution of krill may deepen during the winter (e.g. Tarling \& Fielding 2016). Although optical satellites (passive) are not able to acquire imagery over light-limited seasons in the high latitudes, deeper swarms would still be less detectable via satellites collecting active data. However, knowledge of the frequency of occurrence of detectable surface swarms over the late spring to early autumn seasons would be valuable information in itself.

The size of a surface swarm that can be detected depends on the spatial resolution of the satellite used. For example, the Visible Infrared Imaging Radiometer Suite (VIIRS) aboard Suomi and the Ocean and Land Colour Instrument (OLCI) aboard Sentinel-3 offer a maximum (full) spatial resolution of $300 \mathrm{~m}$, making the detection of smaller swarms challenging. However, large surface patches of red zooplankton that have previously been detected using VIIRS covered several thousand kilometres (Basedow et al. 2019). The length of krill swarms vary; Tarling et al. (2009) classified swarms into different types, with mean swarm lengths ranging from $26 \mathrm{~m}$ for small swarms to $1174 \mathrm{~m}$ for super-swarms. Fielding et al. (2012) measured a mean swarm length of 70-94 $\mathrm{m}$ between spring and autumn (maximum length $=4255 \mathrm{~m}$, minimum length $=30 \mathrm{~m}$ ). The depth of a krill swarm and packing concentration will affect how much of a pixel needs to contain krill to retain spectral information about krill. The high resolution $(10 \mathrm{~m})$ data collected by Sentinel-2 offer appropriate resolution for detecting small surface swarms of krill in this region; however, the absence of spectral bands at $~ 580$ and $625 \mathrm{~nm}$ must also be considered. DigitalGlobe's WorldView satellites launched from 2009 may be valid contenders for swarm detection. These satellites collect data in the bands corresponding to the three krill peaks at $450-460,580-590$ and $625 \mathrm{~nm}$, and at even higher spatial resolutions $(1.8 \mathrm{~m})$ than Sentinel-2. Unfortunately, these commercial data are not provided for free and require planned image collection, so they would not be as suitable to scan for swarms. The planned future hyperspectral satellite, EnMAP, will provide increased spectral resolution, with continuous bands of 6-14 nm width, sampled at $5-12 \mathrm{~nm}$ intervals, which is particularly suitable for detecting distinguishing features at any part of the spectrum. Additionally, the planned NASA PACE mission will have high spectral resolution ( $5 \mathrm{~nm}$ intervals from 340 to $890 \mathrm{~nm}$ ), which would be extremely useful, but the limited spatial resolution ( $1 \mathrm{~km}$ per pixel) may be too coarse to detect the majority of krill swarms. Future studies looking to use satellite remote sensing for krill detection probably need to focus on the unique peaks we have identified $(450-460,580-590$ and $625 \mathrm{~nm})$ and to ensure that the selected satellites retrieve data in these bands.

The ability to detect krill swarms remotely depends on multiple properties of the krill swarm, as well as other constituents in the water affecting the optical depth and the spatial and spectral resolution of the remote sensor. Therefore, currently it would not be possible to resolve any vertical swarm structure and measure the concentration of krill remotely. The main benefit would be in identifying the size and location of large swarms at the surface, which, combined with knowledge about these swarms (e.g. Tarling et al., 2009), could be used to estimate krill concentration.

Finally, it is important to consider the spectral properties of other constituents in the water column and whether other particles could impart a similar signal to krill. Whilst other organisms exist that contain similar pigments (e.g. other species of krill), Antarctic krill are numerically abundant and known swarmers, thus they are likely to be the dominant cause of red colouration in the surface on the scales needed to be detected by Sentinel-3, with a spatial resolution of $300 \mathrm{~m}$. It is probable that, as has been suggested for the application of lidar to detect zooplankton (Churnside \& Thorne 2005), successful remote detection would require the survey area to be dominated by a single zooplankton type to prevent a mixed spectral signature. Densely packed krill swarms are thus obvious candidates for remote detection. Co-location of krill with, for example, very high chlorophyll might affect our ability to detect swarms, in part due to the reduced optical depth resulting from high surface phytoplankton concentrations. Further field studies are required to assess whether very high 
chlorophyll concentrations could outweigh the reflectance signal of krill and thus prevent their remote detection in such regions.

\section{Remote detection of krill}

The power of satellites is that they are able to measure over large synoptic scales for most of the year. At very high latitudes, the lack of incoming light in winter prevents the collection of ocean colour remote sensing data by passive sensors. Active lidar sensors such as the Cloud-Aerosol Lidar with Orthogonal Polarization (CALIOP) aboard CALIPSO have been used to examine diel vertical migration in copepods using backscatter (Behrenfeld et al. 2019). This method enables both day and night data collection and hence includes the polar winter. However, while lidar remote sensors can measure at night and to greater depths, these sensors cannot penetrate cloud. Thus, in the Southern Ocean, cloud cover is likely to present a continued challenge for the regular detection of swarms over large scales. This also presents a challenge for future targeted direct validation and ground truthing, as in situ measurements will need to coincide with cloud-free conditions for satellite measurements. Direct validation is further limited as employed in situ measurements (nets and acoustics) often do not provide information about krill in the upper $15 \mathrm{~m}$ of the water column. An additional barrier to satellite detection is related to strong storms leading to a dominance of white caps and poor-quality reflectance data. Detection of krill from satellite images may therefore require some sophisticated search algorithms and is not likely to be synoptically comprehensive. Analysis of areas where detection may be possible may nevertheless allow environmental relationships to be established for wider spatial and temporal extrapolations.

Considering the aforementioned limitations, we suggest that additional field campaigns be conducted to validate our findings and achieve unambiguous remote detection of krill surface swarms. Specifically, we suggest a survey campaign utilizing deck observers, surface nets and multispectral, hyperspectral or lidar sensors mounted on the ship and on unmanned aerial vehicles (UAVs)/drones. These data need to be collected near to satellite overpass times when there are gaps in clouds, wherever possible, to evaluate the use of satellites for remote detection. Through a combination of acoustic measurements and deck observations, surface krill swarms can be targeted and sampled with net tows. These net tows should follow the procedures of the Stramin net tows of the Discovery Investigations (Marr 1962), where the net is towed abeam of the ship to try and minimize net avoidance during the day. UAVs/drones mounted with appropriate sensors can be flown in a survey pattern over the region, which, combined with the ship-mounted spectral sensors, will obtain cloud-free reflectance imagery of the surface krill swarms at a high spatial resolution. Churnside \& Thorne (2005) compared airborne lidar and acoustic data to examine zooplankton in the upper $24 \mathrm{~m}$ and found a good correlation, although appropriate thresholds were needed to remove the effects of smaller particles such as sediments and phytoplankton, and backscatter coefficients must be known in order to obtain quantitative data. With successful ground truthing and validation, an airborne approach (rather than satellite) can enable a more wide-scale assessment of krill using remote spectral measurements, such as by mounting sensors on regular operational flights to and from Antarctica or by regular drone surveys from research stations.

\section{Conclusion}

This study provides the first reflectance spectra of freshly caught Antarctic krill, information that is necessary for remote detection such as via satellites. We find that the reflectance spectra of krill are significantly different from that of ocean water and show a strong absorbance feature that probably corresponds to the presence of the pigment astaxanthin. We suggest that the next steps towards the remote detection of krill require further field campaigns utilizing spectral measurements from UAVs and daytime surface net tows. A remote detection capability will improve ongoing assessments of krill distributions and biomass, thus supporting sustainable fisheries management and understanding of the impacts of krill on biogeochemical cycles.

\section{Acknowledgements}

A big thank you is given to all of the crew and scientists aboard the RRS James Clark Ross during JR19001 for their hard work, good humour and help in keeping the krill incubation facility (the 'Krillton') open for business. We thank David McKee for advice at the early stages of the project. We gratefully acknowledge the support of the Natural Environment Research Council Field Spectroscopy Facility (NERC FSF) for the loan of equipment required to carry out this research. Additionally, we thank the two anonymous reviewers for taking the time to review our manuscript.

\section{Financial support}

This work was made possible by a bursary awarded to Anna Belcher from Antarctic Science Ltd. Sophie Fielding, Geraint A. Tarling, Gabriele Stowasser and Anna Belcher were supported by the BAS Ecosystems programme. Peter Fretwell and Louise Ireland were funded through NERC core funding, Andrew Gray by the NERC FSF and Lauren Biermann through the 
NERC Earth Observation Data Acquisition and Analysis Service (NEODAAS).

\section{Author contributions}

$\mathrm{AB}$ and GAT devised the project and planned the experiments with the help of PF and AG. AB, GS and SF carried out field sampling of krill and $A B$ carried out on-board laboratory experiments. LI and LB provided expertise on satellite data. $\mathrm{AB}$ wrote the manuscript with support from all authors.

\section{Details of data deposit}

Raw spectroradiometer data are housed with the Polar Data Centre: Belcher, A. (2021). Antarctic krill reflectance measurements - spectroradiometer data (Version 1.0) [dataset]. NERC EDS UK Polar Data Centre; https://doi. org/10.5285/54643342-F2ED-43F4-ADA2-87E81181FF88.

\section{Supplemental material}

A supplemental figure will be found at https://doi.org/10. 1017/S0954102021000262.

\section{References}

ASD, Inc. 2010. FieldSpec ® 3 user manual. Boulder, CO, ASD, Inc., $98 \mathrm{pp}$.

Atkinson, A., Siegel, V., Pakhomov, E.A., Jessopp, M.J. \& Loeb, V. 2009. A re-appraisal of the total biomass and annual production of Antarctic krill. Deep-Sea Research I: Oceanographic Research Papers, 56, 10.1016/j.dsr.2008.12.007.

Atrinson, A., Hill, S.L., Pakhomov, E.A., Siegel, V., Reiss, C.S., Loeb, V.J., et al. 2019. Krill (Euphausia superba) distribution contracts southward during rapid regional warming. Nature Climate Change, 9, 10.1038/s41558-018-0370-z.

Auerswald, L., Freier, U., Lopata, A. \& Meyer, B. 2008. Physiological and morphological colour change in Antarctic krill, Euphausia superba: a field study in the Lazarev Sea. Journal of Experimental Biology, 211, 10.1242/jeb.024232.

Basedow, S.L., McKee, D., Lefering, I., Gislason, A., Daase, M., TrudnowsKa, E., et al. 2019. Remote sensing of zooplankton swarms. Scientific Reports, 9, 10.1038/s41598-018-37129-x.

Behrenfeld, M.J., Gaube, P., Della Penna, A., O'Malley, R.T., Burt, W.J., Hu, Y., et al. 2019. Global satellite-observed daily vertical migrations of ocean animals. Nature, 576, 10.1038/ s41586-019-1796-9.

Belcher, A., Henson, S.A.A., Manno, C., Hill, S.L.L., Atrinson, A., THORPE, S.E.E., et al. 2019. Krill faecal pellets drive hidden pulses of particulate organic carbon in the marginal ice zone. Nature Communications, 10, 10.1038/s41467-019-08847-1.

Cavan, E.L., Belcher, A., Atkinson, A., Hill, S.L., Kawaguchi, S., McCormack, S., et al. 2019. The importance of Antarctic krill in biogeochemical cycles. Nature Communications, 10, 10.1038/ s41467-019-12668-7.

Churnside, J.H. \& Thorne, R.E. 2005. Comparison of airborne lidar measurements with $420 \mathrm{kHz}$ echo-sounder measurements of Zooplankton. Applied Optics, 44, 10.1364/AO.44.005504.
Clarke, A. \& Tyler, P.A. 2008. Adult Antarctic krill feeding at abyssal depths. Current Biology, 18, 10.1016/j.cub.2008.01.059.

Cubaynes, H.C., Fretwell, P.T., Bamford, C., Gerrish, L. \& Jackson, J.A. 2019. Whales from space: four mysticete species described using new VHR satellite imagery. Marine Mammal Science, 35, 10.1111/ mms. 12544 .

Czerpak, R., JACKOWSKA, H. \& Mical, A. 1980. Qualitative analysis of carotenoids in particular parts of body of males and females of Euphausia superba Dana (Crustacea). Polish Polar Research, 1, 139-145.

Davies, E.J., Basedow, S.L. \& McKeE, D. 2021. The hidden influence of large particles on ocean colour. Scientific Reports, 11, 10.1038/ s41598-021-83610-5.

Dierssen, H.M. \& Randolph, K. 2013. Remote sensing of ocean color. In ORCuTt, J., ed. Earth system monitoring: selected entries from the Encyclopedia of Sustainability Science and Technology. New York: Springer New York, 439-472.

Everson, I. \& Bone, D.G. 1986. The effectiveness of the RMT8 system for sampling krill (Euphausia superba) swarms. Polar Biology, 6, 10.1007/BF00258257.

Fielding, S., Watkins, J.L., Collins, M.A., Enderlein, P. \& Venables, H.J. 2012. Acoustic determination of the distribution of fish and krill across the Scotia Sea in spring 2006, summer 2008 and autumn 2009. Deep-Sea Research II: Topical Studies in Oceanography, 59-60, 10.1016/j.dsr2.2011.08.002.

Fielding, S., Watkins, J.L., Trathan, P., Enderlein, P., Waluda, C.M., StOWASSER, G., et al. 2014. Interannual variability in Antarctic krill (Euphausia superba) density at South Georgia, Southern Ocean: 1997-2013. ICES Journal of Marine Science, 71, 10.1093/icesjms/fsu104.

HAMNER, W.M. \& HAMNER, P.P. 2000. Behavior of Antarctic krill (Euphausia superba): schooling, foraging, and antipredatory behavior. Canadian Journal of Fisheries and Aquatic Sciences, 57, 10.1139/f00-195

Hewitt, R.P., Watkins, J., Naganobu, M., Sushin, V., Brierley, A.S., Demer, D., et al. 2004. Biomass of Antarctic krill in the Scotia Sea in January/February 2000 and its use in revising an estimate of precautionary yield. Deep-Sea Research II: Topical Studies in Oceanography, 51, 10.1016/j.dsr2.2004.06.011.

IOCCG. 2010. Atmospheric correction for remotely-sensed ocean-colour products. In WANG, M., ed. Reports of the International Ocean-Colour Coordinating Group, No. 10. Dartmouth: IOCCG, 10.25607/OBP-101.

Klevjer, T.A., TARling, G.A. \& Fielding, S. 2010. Swarm characteristics of Antarctic krill Euphausia superba relative to the proximity of land during summer in the Scotia Sea. Marine Ecology Progress Series, 409, 10.3354/meps08602.

Korb, R.E., Whitehouse, M.J., Ward, P., Gordon, M., Venables, H.J. \& Poulton, A.J. 2012. Regional and seasonal differences in microplankton biomass, productivity, and structure across the Scotia Sea: Implications for the export of biogenic carbon. Deep-Sea Research II: Topical Studies in Oceanography, 59-60, 10.1016/ j.dsr2.2011.06.006.

Kwon, E., Primeau, F. \& Sarmiento, J. 2009. The impact of remineralization depth on the air-sea carbon balance. Nature Geoscience, 2, 630-635.

Lee, Z., Wei, J., Shang, Z., Garcia, R., Dierssen, H., Ishizaka, J. \& CASTAGNA, A. 2019. On-water radiometry measurements: skylight-blocked approach and data processing (appendix to IOCCG Protocol Series 2019). Protocols for Satellite Ocean Colour Data Validation: In Situ Optical Radiometry, 3, 1-7.

Macaulay, G., Skaret, G., Knutsen, T., Bergstad, O.A., Krafft, B., FIELDING, S., et al. 2019. Biomass results from the International Synoptic Krill Survey in Area 48, 2019. CCAMLR meeting report. Hobart: Convention for the Conservation of Antarctic Marine Living Resources. 
MARR, J. 1962. The natural history and geography of the Antarctic krill, Euphausia superba Dana. Discovery Reports, 32, 33-464.

Mobley, C.D. 1999. Estimation of the remote-sensing reflectance from above-surface measurements. Applied Optics, 38, 10.1364/ao.38.007442.

NiCOL, S. 1991. CCAMLR and its approaches to management of the krill fishery. Polar Record, 27, 10.1017/S0032247400012626.

NiCOL, S. \& FOSTER, J. 2016. The fishery for antarctic krill: its current status and management regime. In Siegel, V., ed. Biology and ecology of Antarctic krill. Advances in polar ecology. Cham: Springer, 387-421.

Nunes, S., Latasa, M., Delgado, M., Emelianov, M., Simó, R. \& Estrada, M. 2019. Phytoplankton community structure in contrasting ecosystems of the Southern Ocean: South Georgia, South Orkneys and western Antarctic Peninsula. Deep-Sea Research I: Oceanographic Research Papers, 151, 10.1016/j.dsr.2019.06.005.

Rees, W.G., Brown, J.A., Fretwell, P.T. \& Trathan, P.N. 2017. What colour is penguin guano? Antarctic Science, 29, 10.1017/S0954102017000190.

Ryabov, A.B. \& Tarling, G.A. 2019. Scaling of size, shape and surface roughness in Antarctic krill swarms. ICES Journal of Marine Science, 76, 10.1093/icesjms/fsz005.

Schmidt, K., Atrinson, A., Steigenberger, S., Fielding, S., Lindsay, M.C.M., Pond, D.W., et al. 2011. Seabed foraging by Antarctic krill: implications for stock assessment, bentho-pelagic coupling, and the vertical transfer of iron. Limnology and Oceanography, 56, 10.4319/ 10.2011.56.4.1411.

SIEGEL, V. 2016. Biology and ecology of Antarctic krill. Cham: Springer, $441 \mathrm{pp}$.

Simard, Y. \& Sourisseau, M. 2009. Diel changes in acoustic and catch estimates of krill biomass. ICES Journal of Marine Science, 66, 10.1093/icesjms/fsp055.
Soja-Wozniak, M., Darecki, M., Wojtasiewicz, B. \& Bradtke, K. 2018. Laboratory measurements of remote sensing reflectance of selected phytoplankton species from the Baltic Sea. Oceanologia, 60, 86-96.

Standing Committee of Analysts. 1983. The determination of chlorophyll a in aquatic environments 1980. In Methods for the examination of waters and associated materials. London: H.M Stationery Office, 1-26.

TARLING, G.A. \& Fielding, S. 2016. Swarming and behaviour in Antarctic krill. In Siegel, V., ed. Biology and ecology of Antarctic krill. Cham: Springer, 279-319.

Tarling, G.A., Thorpe, S.E., Fielding, S., Klevjer, T., Ryabov, A. \& Somerfield, P.J. 2018. Varying depth and swarm dimensions of open-ocean Antarctic krill Euphausia superba Dana, 1850 (Euphausiacea) over diel cycles. Journal of Crustacean Biology, 38, 10.1093/jcbiol/ruy040.

Tarling, G.A., Klevjer, T., Fielding, S., Watkins, J., Atkinson, A., Murphy, E., et al. 2009. Variability and predictability of Antarctic krill swarm structure. Deep-Sea Research I: Oceanographic Research Papers, 56, 10.1016/j.dsr.2009.07.004.

Tichy, F.E., Solli, H. \& Klaveness, H. 2003. Non-linear effects in a $200-\mathrm{kHz}$ sound beam and the consequences for target-strength measurement. ICES Journal of Marine Science, 60, 571-574.

WatKIns, J.L. 2000. Aggregation and vertical migration. In Everson, I., ed. Krill biology, ecology and fisheries. Oxford: Blackwell Science, 80-102.

Xi, H., Hieronymi, M., Krasemann, H. \& Röttgers, R. 2017. Phytoplankton group identification using simulated and in situ hyperspectral remote sensing reflectance. Frontiers in Marine Science, 4, 10.3389/fmars.2017.00272. 